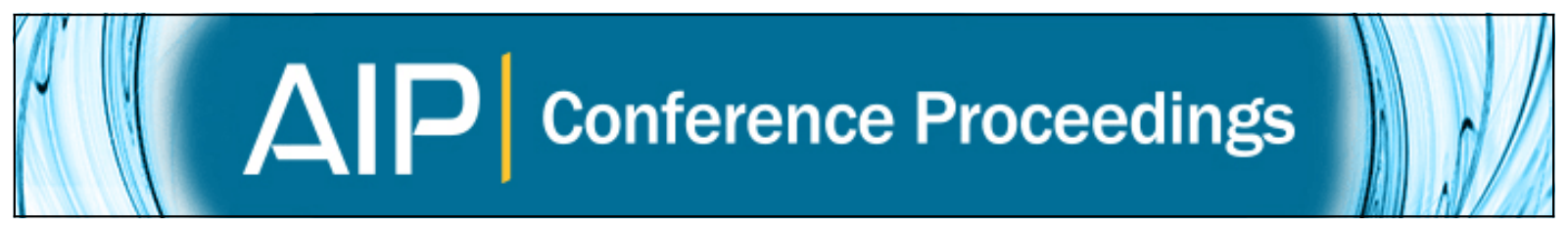

\title{
RF heating for fusion product studies
}

T. Hellsten, T. Johnson, S. E. Sharapov, V. Kiptily, J. Eriksson, M. Mantsinen, M. Schneider, F. Rimini, M. Tsalas , and JET contributors

Citation: AIP Conference Proceedings 1689, 060007 (2015); doi: 10.1063/1.4936505

View online: http://dx.doi.org/10.1063/1.4936505

View Table of Contents: http://scitation.aip.org/content/aip/proceeding/aipcp/1689?ver=pdfcov

Published by the AIP Publishing

\section{Articles you may be interested in}

Study of Heating and Fusion Power Production in ITER Discharges

AIP Conf. Proc. 1392, 92 (2011); 10.1063/1.3647235

Enhanced loss of fusion products during mode conversion heating in TFTR

AIP Conf. Proc. 355, 67 (1996); 10.1063/1.49518

RF Heating Studies on BCTX

AIP Conf. Proc. 244, 65 (1992); 10.1063/1.41651

Fusion applications of rf accelerators

AIP Conf. Proc. 210, 660 (1990); 10.1063/1.39639

Production of Net Fusion Energy from Laser-Heated Target Plasmas

J. Appl. Phys. 42, 1035 (1971); 10.1063/1.1660138 


\title{
RF Heating for Fusion Product Studies
}

\author{
T. Hellsten ${ }^{1, \text { a) }}$ T. Johnson ${ }^{1}$, S. E. Sharapov ${ }^{2}$, V. Kiptily ${ }^{2}$ J. Eriksson ${ }^{3}$, \\ M. Mantsinen ${ }^{4}$, M. Schneider ${ }^{5}$, F. Rimini ${ }^{2}$ M. Tsalas ${ }^{6}$ and JET contributors ${ }^{*}$ \\ ${ }^{1}$ EUROfusion Consortium, JET, Culham Science Centre, Abingdon, OX14 3DB, UK \\ ${ }^{I}$ Dept. of Fusion Plasma Physics, School of Electrical Engineering, KTH, Stockholm, Sweden \\ ${ }^{2}$ CCFE Fusion Association, Culham Science Centre Abingdon, UK \\ ${ }^{3}$ Dept. of Physics and Astronomy, Uppsala University, Sweden \\ ${ }^{4}$ Catalan Institution for Research and Advanced Studies, Barcelona, Spain \\ ${ }^{5}$ IRFM-CEA, Saint-Paul-Lez Durance, France \\ ${ }^{6}$ FOM Inst. DIFFER, Nieuwegein, Netherlands, \\ "See the Appendix of F. Romanelli et al., Proceedings of the $24^{\text {th }}$ IAEA Fusion Energy Conference 2014, \\ Saint Petersburg, Russia \\ a) Corresponding author: thel@kth.se
}

\begin{abstract}
Third harmonic cyclotron heating is an effective tool for accelerating deuterium (D) beams to the MeV energy range, suitable for studying ITER relevant fast particle physics in plasmas without significant tritium content. Such experiments were recently conducted in JET with an ITER like wall in D plasmas with ${ }^{3} \mathrm{He}$ concentrations up to $30 \%$ in order to boost the fusion reactivity by $\mathrm{D}^{3} \mathrm{He}$ reactions. The harmonic cyclotron heating produces high-energy tails in the $\mathrm{MeV}$ range of $\mathrm{D}$ ions by on-axis heating and of ${ }^{3} \mathrm{He}$ ions by tangential off-axis heating. The discharges are characterized by long sawtooth free periods and a rich spectrum of MHD modes excited by the fast $\mathrm{D}$ and ${ }^{3} \mathrm{He}$ ions. The partitions of the power, which depend on the distribution function of D, vary strongly over several slowing down times. Selfconsistent modelling of the distribution function with the SELFO-light code are presented and compared with experimental data from fast particle diagnostics.
\end{abstract}

\section{INTRODUCTION}

$\mathrm{RF}$ heating at the third harmonic cyclotron resonance, $\omega=3 \omega_{\mathrm{CD}}$, is an effective method for accelerating deuterium (D) beams to the MeV energy range [1,2] suitable for studying ITER relevant fast particle physics in plasmas without significant tritium content. Such experiments were conducted recently in the JET tokamak with an ITER like wall in D plasmas with ${ }^{3} \mathrm{He}$ concentrations up to $30 \%$. The discharges are characterized by long sawtoothfree periods and a rich spectrum of MHD modes excited by the fast ions. RF heating at higher frequencies is often used for fast wave current drive experiments because of the weaker cyclotron absorption at harmonic resonances [35]. However, since harmonic cyclotron absorption is sensitive to the distribution function, off-axis heating may still absorb significant power if high-energy tails are formed. In the experiments for fusion product studies reported here, in order to enhance the fusion yield by D-D and D- ${ }^{3} \mathrm{He}$ reactions, the frequency and the magnetic field were chosen such that resonance $\omega=3 \omega_{\mathrm{CD}}$ passed close to the magnetic axis, $R_{0}=3 \mathrm{~m}$. The second harmonic resonance of ${ }^{3} \mathrm{He}$, $\omega=2 \omega_{\mathrm{C} 3 \mathrm{He}}$, appeared then at $0.32 \mathrm{~m}$ off-axis at $R=2.68 \mathrm{~m}$ near the $q=1$ surface. The off-axis heating of ${ }^{3} \mathrm{He}$ produced a strongly localised high-energy tail where the resonance tangents the flux surface. Because of the enhanced third harmonic $\mathrm{D}$ heating the power absorption by ${ }^{3} \mathrm{He}$ became low. 


\section{SIMULATIONS OF JET EXPERIMENTS}

The ion distribution functions were calculated with the SELFO-light code [6], in which the equilibrium, calculated with the CHEASE code [7], is separated into a set of shells limited by magnetic flux surfaces, here denoted as subvolumes. The wave field is calculated with the LION code $[8,9]$ and the time dependent distribution functions with a 1D Fokker-Planck code [10]. The dielectric tensors in LION are defined from local homogeneous bi-Maxwellian susceptibility tensors to all orders in ion Larmor radius to the magnetosonic wavelength. In order to include the effects of non-thermal distribution functions caused by ion cyclotron heating the parallel ion temperatures and the susceptibility tensor elements are modified by flux surface averaged amplification factors [6]. The electron damping caused by ELD/TTMP is calculated for a local homogeneous plasma, and added to the antiHermitian part of the electron susceptibility tensor [6]. The flux surface averaged distribution functions are calculated for each subvolume and resonant ion species with the Fokker-Planck code by averaging the wave field and quantities obtained from the wave solver over the subvolumes.

In order to improve the modelling for medium sized tokamaks as the widths of the drift orbits become comparable with the minor radius of the plasma the SELFO-light code has been upgraded by including an orbit solver, which is used for averaging the collision operators in the Fokker-Planck code over drift orbits. For the 1D Fokker-Planck solver we assume when a cyclotron resonance or harmonics intersects the subvolume that the orbits are trapped and have their turning points at the resonance, otherwise the orbits are assumed to be thin passing ones. If more than one harmonic intersect the orbit, a choice has to be made about the relevant one. Self-consistent modelling is obtained by means of iterations such that the absorbed power calculated from the wave field is consistent with that calculated with the quasi-linear operator in the Fokker-Planck solver.

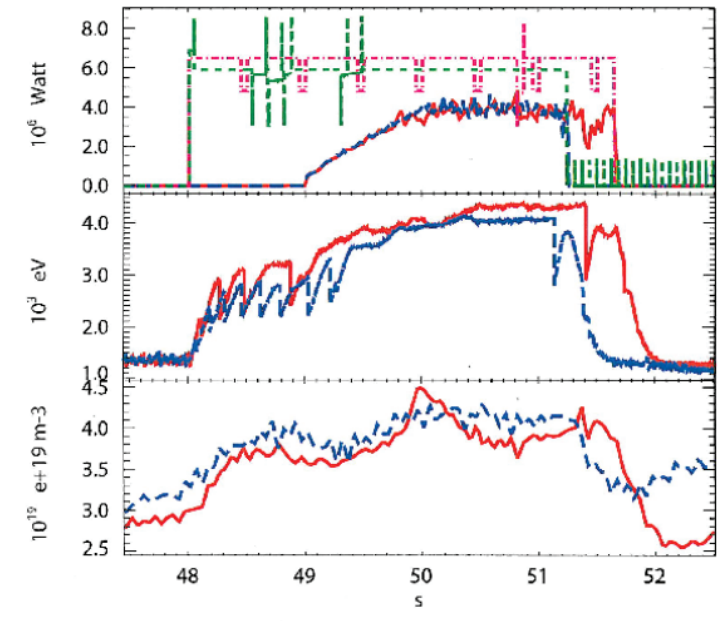

FIGURE 1. Time traces for \#86775 (red full line and cerise dashed-dotted) and \#86779 (blue and green dashed lines) (top) NBI and RF powers, (middle) central electron temperature, (bottom) central electron density

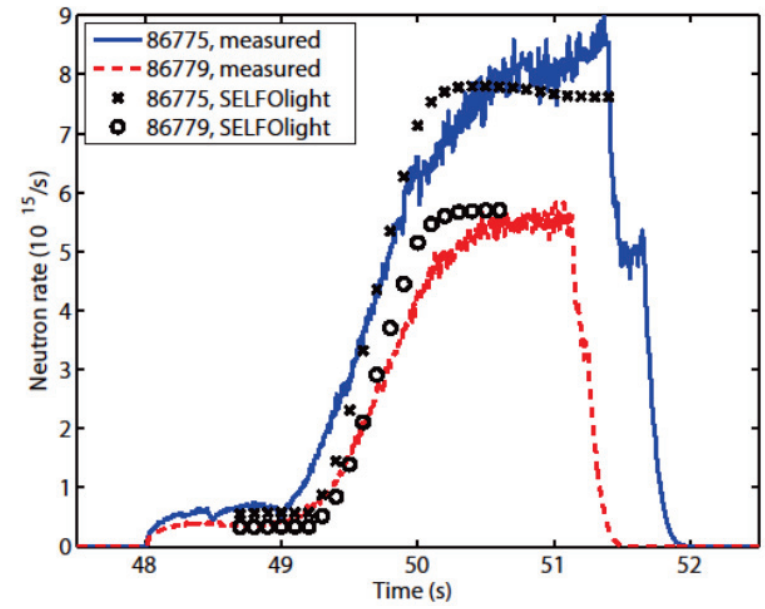

FIGURE 2. Neutron rate versus time \#86775 and \#86779.

The discharges analysed here had a vacuum magnetic field of $2.24 \mathrm{~T}$ at $R=3.0 \mathrm{~m}$, a plasma current of $1.8 \mathrm{MA}$, they were heated with NBI at $100 \mathrm{kV}$ and with $51 \mathrm{MHz}$ RF waves coupled with the antennas in dipole phasing. Time traces of the discharges \#86775 and \#86779 with about $13 \%$ and $20 \%{ }^{3} \mathrm{He}$, respectively, are shown in Fig. 1 . When modelling the discharges the density and temperature profiles were assumed to be constant in time, the NBI source term was calculated with the PENCIL code. The distribution function for NBI heating alone becomes stationary after $\sim 0.1 \mathrm{~s}$. A spectrum of five toroidal modes was used to simulate the RF-heating: $P\left(n_{\phi}=18\right) / \mathrm{P}_{\text {tot }}=0.05$, $P\left(n_{\phi}=20\right) / \mathrm{P}_{\text {tot }}=0.25, P\left(n_{\phi}=27\right) / \mathrm{P}_{\text {tot }}=0.40, P\left(n_{\phi}=31\right) / \mathrm{P}_{\text {tot }}=0.25$, and $P\left(n_{\phi}=46\right) / \mathrm{P}_{\text {tot }}=0.05$. The following parameters were used for \#86775: $n_{\mathrm{e} 0}=5.1 \times 10^{19} \mathrm{~m}, n_{\mathrm{D} 0}=3.17 \times 10^{19} \mathrm{~m}^{-3}, n_{\mathrm{He} 0}=0.66 \times 10^{19} \mathrm{~m}^{-3}, n_{\mathrm{C} 0}=0.12 \times 10^{19} \mathrm{~m}^{-3}, T_{\mathrm{e} 0}=4.4 \mathrm{keV}, T_{\mathrm{i} 0}=$ $2.1 \mathrm{keV}$, a neutral beam power of $6.5 \mathrm{MW}$, the RF-power followed the experimental one with a $1 \mathrm{~s}$ linear ramp and a flat top of 3.7MW for $2.0 \mathrm{~s}$. The discharge $\# 86779$ had: $n_{\mathrm{e} 0}=5.3 \times 10^{19} \mathrm{~m}^{-3}, n_{\mathrm{D} 0}=2.44 \times 10^{19} \mathrm{~m}^{-3}, n_{\mathrm{He} 0}=1.06 \times 10^{19} \mathrm{~m}^{-3}$, $n_{\mathrm{C} 0}=0.12 \times 10^{19} \mathrm{~m}^{-3}, T_{\mathrm{e} 0}=4.2 \mathrm{keV}, T_{\mathrm{i} 0}=3.38 \mathrm{keV}$, a neutral beam power of $5.9 \mathrm{MW}$ and a RF flat top power of $4.0 \mathrm{MW}$. 
The dominant absorption mechanisms for the discharges described above are $\omega=3 \omega_{\mathrm{CD}}, \omega=2 \omega_{\mathrm{C} 3 \mathrm{He}}$ and TTMP/ELD, the latter two dominate for thermal plasmas. The TTMP/ELD dominates the upper part $\left(n_{\phi}=31\right.$ and $\left.n_{\phi}=46\right)$ of the toroidal mode spectrum and $\omega=2 \omega_{\mathrm{C} 3 \mathrm{He}}$ the lower part $\left(n_{\phi}=18\right.$ and $\left.n_{\phi}=20\right)$. The partitions of absorbed RF power are shown in Table 1. In the first row the partitions are given for thermal plasmas, in the second row for NBI heated plasmas at the onset of the RF power and in the third row for the steady state with NBI and RF, which occurred at about $0.4 \mathrm{~s}$ after the RF-power reached the flat top. Because of the low damping by the third harmonic heating the NBI is essential for preheating and to increase the damping by beam ions. The presence of ${ }^{3} \mathrm{He}$ does not only increase the fusion power due to $\mathrm{D}-{ }^{3} \mathrm{He}$ reactions, but it also improves the coupling by increasing the single pass damping, in particular for modes belonging to

TABLE 1

\begin{tabular}{|l|r|l|l|l|}
\hline & Discharge & $\begin{array}{l}\boldsymbol{P}_{\mathrm{D}} / \\
\boldsymbol{P}_{\text {tot }}\end{array}$ & $\begin{array}{l}\boldsymbol{P}_{\text {He }} / \\
\boldsymbol{P}_{\text {tot }}\end{array}$ & $\begin{array}{l}\boldsymbol{P}_{\text {ed }} / \\
\boldsymbol{P}_{\text {tot }}\end{array}$ \\
\hline no & 86775 & 0.11 & 0.29 & 0.60 \\
NBI & 86779 & 0.07 & 0.38 & 0.55 \\
\hline with & 86775 & 0.24 & 0.24 & 0.52 \\
NBI & 86779 & 0.18 & 0.36 & 0.46 \\
\hline steady & 86775 & 0.82 & 0.03 & 0.15 \\
state & 86779 & 0.83 & 0.04 & 0.13 \\
\hline
\end{tabular}
the lower part of the spectrum (for $n_{\phi}=18-20$, in absence of beams, the single pass damping doubled with ${ }^{3} \mathrm{He}$, and with beams it increased with about $30 \%$ ).
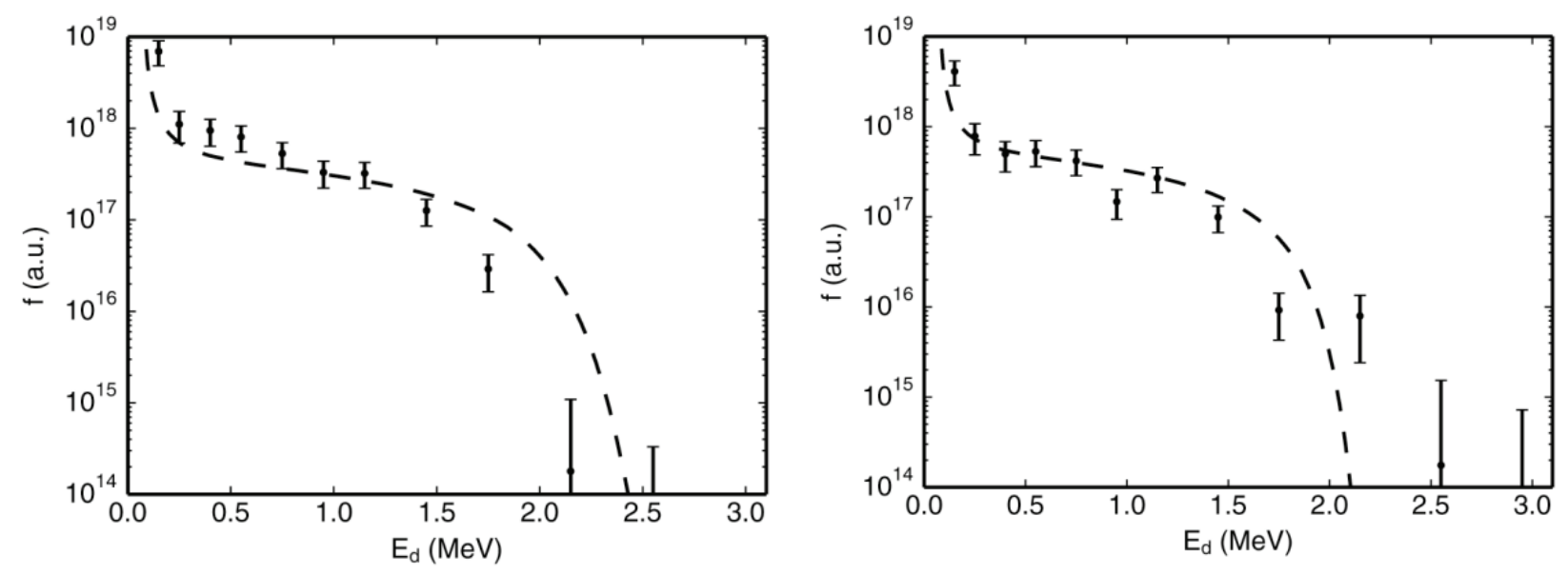

FIGURE 3. Deuterium spectra left \#86775 and right \#86779, dashed line calculations and • measurements.

In order to validate the SELFO-light code we compare in Fig. 2 the measured neutron rates with the calculated ones with cross-sections given in Ref. [11]. In Fig. 3 the energy spectra of the deuterium deduced from the TOFOR neutron spectrometer [12] are compared with the simulations; the distribution function of deuterium extends up to the first zeroth of the quasi-linear RF-operator. The fusion rate from $\mathrm{D}-{ }^{3} \mathrm{He}$ reactions were for $\# 867750.44 \times 10^{16} \mathrm{~s}^{-1}$ and for $\# 867790.75 \times 10^{16} \mathrm{~s}^{-1}$, corresponding to $13 \mathrm{~kW}$ and $21 \mathrm{~kW}$, respectively. The experimental ones, deduced from gamma rays, gave about $10 \mathrm{~kW}$ of power. Initially the ${ }^{3} \mathrm{He}$ ions absorbed a significant fraction of the power, as the D absorption increases the ${ }^{3} \mathrm{He}$ absorption diminishes. The ${ }^{3} \mathrm{He}$ absorption is strongly localised, $2.665 \mathrm{~m}<R<2.695 \mathrm{~m}$ around the flux surface that tangents the cyclotron resonance, which width is consistent with the Doppler broadening of the cyclotron resonance of thermal ${ }^{3} \mathrm{He}$ ions. The strongest tail was obtained $0.5 \mathrm{~s}$ after the onset of the RF-power with the high-energy tail extending up to $2 \mathrm{MeV}$. The contribution from the fast ${ }^{3} \mathrm{He}$ ions to the fusion rate is negligible. The presence of high energy ${ }^{3} \mathrm{He}$ ions with the turning points near $\omega=2 \omega_{\mathrm{C} 3 \mathrm{He}}$ is consistent with the measurements with the scintillator probe. The total energy content measured with the diamagnetic loop is for \#86775 2.5MJ. The calculated thermal energy and $3 / 2$ off the fast energy, $0.47 \mathrm{MJ}$, give a total of $2.4 \mathrm{MJ}$. For discharge $\# 86779$ the corresponding measured diamagnetic energy is $2.3 \mathrm{MJ}$ and the corresponding total calculated energy is $2.2 \mathrm{MJ}$ of which the fast energy constitutes $0.43 \mathrm{MJ}$. 


\section{CONCLUSIONS AND DISCUSSIONS}

The SELFO-light code has been validated against RF heating experiments at $\omega=3 \omega_{\mathrm{CD}}$ in JET. The neutron rate, which increases with more than an order of magnitude with the RF-power, is reasonable modelled taken into account that the power partition between the different species changes strongly due to the built up of high-energy tails. The assumptions of constant temperatures and densities are reasonable satisfied for \#86779. For \#86775 the density increases before the sawtooth crash at $t=51.4 \mathrm{~s}$ resulting in a larger disagreement of the time behaviour of the fusion rate. Also the energy spectra of the deuterium deduced from the neutron spectrometer and the measured diamagnetic energies agree within the error bars with the simulated ones. The fusion rates are sensitive to the temperature profiles, and the error bars in the ion temperature profiles and ${ }^{3} \mathrm{He}$ concentration are significant. The modelled neutron rates do not include beam-beam collisions. Overestimation of the ${ }^{3} \mathrm{He}$ concentration and neglect of fast particle losses by TAEs may explain the higher $\mathrm{D}-{ }^{3} \mathrm{He}$ rate. The power partition varied strongly during the heating phase. However, at the last second of the RF-power flat top the power partition was not very sensitive to the parameters, because the increased absorption by $\omega=3 \omega_{\mathrm{CD}}$ resulted that nearly all power was absorbed by the deuterium. In these simulation no losses of RF power e.g. due to RF sheath has been assumed, which would be more important in the early heating phase when the single pass damping is low.

\section{ACKNOWLEDGMENTS}

This work has been carried out within the framework of the EUROfusion Consortium and has received funding from the Euratom research and training programme 2014-2018 under grant agreement No 633053. The views and opinions expressed herein do not necessarily reflect those of the European Commission.

\section{REFERENCES}

1. L.-G. Eriksson et al., Nucl. Fusion (1998)

2. M. Mantsinen et al., Phys. Rev. Lett. 88, 105002 (2002)

3. C. C. Petty et al Nuclear Fusion 39 1421(1999)

4. G. Taylor et al Radio Frequency Powers in Plasmas Proc. 19th Topical Conf. New Port (2011) 325

5 A. Hannan et al Nuclear Fusion 53043005 (2013)

6. T. Hellsten et al Nuclear Fusion 53093004 (2013)

7. H. Lütjens et al., Comput. Phys. Commun. 97 (1996) 219

8. L.Villard et al., Computer Physics Reports 4 (1986) 95.

9. L. Villard et al., Nuclear Fusion 35 (1995) 1173.

10. A. Hannan et al., EPS 2011 Strassbourg, France P5 125

11. H.-S. Bosch and G. M. Hale, Nuclear Fusion 32 (1992) 611

12. C. Hellesen et al, Nuclear Fusion 53 (2013) 11300. 